PROCEEDINGS OF THE

AMERICAN MATHEMATICAL SOCIETY

Volume 129, Number 11, Pages 3413-3416

S 0002-9939(01)05931-7

Article electronically published on May 3, 2001

\title{
ON UNIFORM PROPERTIES OF DOUBLING MEASURES
}

\author{
MICHAEL RUZHANSKY \\ (Communicated by Christopher D. Sogge)
}

\begin{abstract}
In this paper we prove that if $(X, d, \mu)$ is a metric doubling space with segment property, then $\inf r(E) / r(B)>0$ if and only if inf $\mu(E) / \mu(B)>$ 0 , where the infimum is taken over any collection $\mathcal{C}$ of balls $E, B$ such that $E \subset B \subset X$. As a consequence we show that if $X$ is a linear metric doubling space, then it must be finite dimensional.
\end{abstract}

Let $X$ be a metric space and let $d$ denote its metric. Let $\mu$ be a measure on $X$. Then $\mu$ is called doubling if there exists a constant $A$ such that for every $x \in X$, $r>0$, and any ball $B=B(x, r)=\{y \in X: d(y, x)<r\}$,

$$
\mu(B(x, r)) \leq A \mu\left(B\left(x, \frac{r}{2}\right)\right)
$$

holds. One also says that $\mu$ satisfies the doubling condition in this case. The doubling measures are used in many areas of analysis. In particular, in [2] and [6], one defines the notion of Sobolev spaces on metric doubling spaces and shows that a generalization of the Poincaré inequality holds. If $X$ is a stratified group, the defined spaces coincide with Folland-Stein Sobolev spaces; see [4]. For some analysis in spaces of homogeneous type see 1]. In [7] the representation formulas are established for the Sobolev spaces of high orders and they are shown to be equivalent to Poincaré inequalities of high order on doubling metric spaces. The expository paper [3] explains the results of [2] and [7]. In [5], the authors develop the distribution theory on doubling metric spaces and define the notion of high order gradients of functions. The analysis can be used to develop a general distribution theory on doubling metric spaces also in the infinite dimensional setting. One of the most interesting cases is the case of linear spaces $X$. However, the presence of a linear structure turns out to be very restrictive for the doubling spaces. We will show that if the space $X$ is linear, then in fact it must be finite dimensional. This also shows that the use of Gelfand triples is essential for linear spaces; see for example 8]. This result (Theorem 1) will follow from a uniform property of doubling measures established in Theorem2. Another application of Theorem2 will appear in 5], where the polynomials of a given degree are constructed in doubling metric spaces with the segment property and the notions of high order gradients are given and representation formulas in terms of high order gradients are derived.

Theorem 1. Let $(X, d)$ be a complete metric linear space with a doubling measure $\mu$, such that $0<\mu(B)<\infty, B$ a unit ball in $X$. Then $X$ is finite dimensional.

Received by the editors November 23, 1999 and, in revised form, March 24, 2000.

2000 Mathematics Subject Classification. Primary 54E35, 54E50, 46A03, 28 E15.

(C)2001 American Mathematical Society 
Note that the theorem implies that any Frechet space with a doubling measure must be finite dimensional. However, because condition (1) depends on a fixed measure, we prefer to view metrizable locally convex spaces as metric spaces with a fixed measure.

For a ball $B$ by $x(B)$ and $r(B)$ we will denote the center and the radius of $B$, respectively. For $x \in X$ and $r>0$ by $S(x, r)=\{y \in X: d(y, x)=r\}$ we will denote the sphere of radius $r$ centered at $x$. Theorem 1 will follow from the following general property of doubling metric spaces with segment property.

Theorem 2. Let $(X, d, \mu)$ be a metric doubling space which satisfies the segment property, i.e. for each pair of points $x, y \in X$, there exists a continuous curve $\gamma=\gamma(t)$ connecting $x$ and $y$ and such that $d(\gamma(t), \gamma(s))=|t-s|$ for all $t$ and $s$.

(i) Let $B$ be a ball in $X$ such that $S(x(B), r(B))$ is not empty. Then for every $0<\alpha<1$ there exists $0<\beta<1$ such that for every ball $E \subset B$ with $\mu(E)>\alpha \mu(B)$ holds $r(E)>\beta r(B)$. On the other hand for every $0<\beta<1$ there exists $0<\alpha<1$ such that for every ball $E \subset B$ with $r(E)>\beta r(B)$ holds $\mu(E)>\alpha \mu(B)$.

(ii) Let $\mathcal{C}$ be a collection of pairs $(E, B)$ of the balls $E, B \subset X$ such that $E \subset B$ and $\partial B$ is not empty. Then $\inf _{\mathcal{C}} r(E) / r(B)>0$ if and only if $\inf _{\mathcal{C}} \mu(E) / \mu(B)>0$.

The segment property appears naturally in many applications (see [2], [5], [7]). First we will establish some general properties of metric doubling spaces.

Lemma 3. Let $(X, d)$ be a metric space and let a measure $\mu$ on $X$ satisfy (1). Let $B(x, r)$ be a ball and let $y \in X$ be such that $d=d(y, x)>r$. Then

$$
\mu(B(x, r+2(d-r))) \geq\left(1+A^{-k}\right) \mu(B(x, r))
$$

for all $k \in \mathbb{N}$ such that $k>\log _{2} \frac{d+r}{d-r}$. We also have

$$
\mu(B(y, d+r)) \geq\left(1+A^{-k}\right) \mu(B(x, r)) .
$$

Proof. By the triangle inequality $B(y, d+r) \supset B(x, r)$ and $\mu(B(y, d+r)) \geq \mu_{0}$ with $\mu_{0}=\mu(B(x, r))$. The doubling property implies

$$
\mu(B(y, d-r)) \geq A^{-k} \mu\left(B\left(y, 2^{k}(d-r)\right)\right.
$$

for every $k$. In particular, $B\left(y, 2^{k}(d-r)\right) \supset B(y, d+r)$ when $k$ is as in the assumption of the lemma. This means $\mu(B(y, d-r)) \geq A^{-k} \mu_{0}$. The balls $B(y, d-r)$ and $B(x, r)$ are disjoint because $z \in B(y, d-r) \cap B(x, r)$ would imply $d(y, x) \leq$ $d(y, z)+d(z, x)<(d-r)+r=d$. By the triangle inequality if $z \in B(y, d-r)$, then

$$
d(x, z) \leq d(x, y)+d(y, z) \leq d+(d-r)=r+2(d-r),
$$

so the ball $B(x, r+2(d-r))$ contains both $B(y, d-r)$ and $B(x, r)$. This implies the statement of the lemma. The last statement is similar.

Proof of Theorem 2, The first statement easily implies the second one. Suppose first that there exists $\alpha \in(0,1)$ such that for every $\beta \in(0,1)$ there exists a ball $E_{\beta} \subset B$ with $\mu\left(E_{\beta}\right)>\alpha \mu(B)$ and $r\left(E_{\beta}\right) \leq \beta r(B)$. Let $m=\left\lceil\log _{1+A^{-2}} \alpha^{-1}\right\rceil$.

Assume first that there exist $a>0$ and $E_{\beta}$ as above such that $d\left(x\left(E_{\beta}\right), x(B)\right)<$ $r(B)-a$ and $r\left(E_{\beta}\right) \leq 3^{-m} a$. We have $3^{m} E_{\beta} \subset B$ since every $x \in 3^{m} E_{\beta}$ satisfies

$$
\begin{aligned}
d(x, x(B)) & \leq d\left(x, x\left(E_{\beta}\right)\right)+d\left(x\left(E_{\beta}\right), x(B)\right) \\
& <3^{m} r\left(E_{\beta}\right)+(r(B)-a) \leq a+(r(B)-a)=r(B) .
\end{aligned}
$$


Let $\gamma$ be a continuous curve connecting $x\left(E_{\beta}\right)$ with a point $z \in S(x(B), r(B))$. Then the length of $\gamma$ is not smaller than $3^{m} r\left(E_{\beta}\right)$, so we can apply Lemma 3 to $E_{\beta}$ with $d=2 r\left(E_{\beta}\right)$. It follows that

$$
\mu(B) \geq \mu\left(3^{m} E_{\beta}\right) \geq\left(1+A^{-2}\right)^{m} \mu\left(E_{\beta}\right) \geq \alpha^{-1} \mu\left(E_{\beta}\right),
$$

which contradicts the choice of $E_{\beta}$. Suppose now that there is no $a$ as above. For $\alpha$ as in the beginning of the proof, we take $\beta=3^{-m} / 2$ and $E_{\beta}$ as above with $r\left(E_{\beta}\right) \leq$ $\beta r(B)=3^{-m} a$ if $a=r(B) / 2$. It follows that $d\left(x\left(E_{\beta}\right), x(B)\right) \geq r(B)-a=r(B) / 2$. Thus, there exists $E_{\beta}$ such that $d\left(x\left(E_{\beta}\right), x(B)\right) \geq r(B) / 2$ and $r\left(E_{\beta}\right) \leq 3^{-m} r(B) / 2$.

Let's denote $E=E_{\beta}$. Let $\gamma$ be a continuous curve connecting $x(E)$ with $x(B)$. Then the length of $\gamma$ is not smaller than $r(B) / 2$ and we apply Lemma 3 with the ball $E, d=2 r(E)$ and $y \in S(x(E), 2 r(E)) \cap \gamma$, which is nonempty due to the continuity of $\gamma$. The last statement of Lemma 3 implies $\mu(B(y, 3 r(E))) \geq\left(1+A^{-2}\right) \mu(E)$. On the other hand $z \in B(y, 3 r(E))$ implies

$$
\begin{aligned}
d(z, x(B)) & \leq d(z, y)+d(y, x(B))<3 r(E)+d(y, x(B)) \\
& =r(E)+d(x(E), x(B))<r(B),
\end{aligned}
$$

since $E \subset B$. This means that $B(y, 3 r(E)) \subset B$. Applying the same argument inductively $m$ times, we get that $E \subset B$ and $\mu(B) \geq\left(1+A^{-2}\right)^{m} \mu(E) \geq \alpha^{-1} \mu(E)$, which is larger than $\mu(B)$. This contradiction proves the first statement of (i).

Conversely, let $E \subset B$ with $r(E)>\beta r(B)$. First we observe that for every $y \in B$ we have $B(y, 2 r(B)) \supset B$. Indeed, $d(y, z) \leq d(y, x(B))+d(x(B), z)<2 r(B)$ for any $z \in B$. Let us denote $c=2 \beta^{-1}$ and let $m$ be the smallest integer $\geq 1-\log _{2} \beta$. If we take $y=x(E)$ it follows that $\operatorname{cr}(E)>2 r(B)$ and $B \subset B(x(E), \operatorname{cr}(E)) \subset$ $B\left(x(E), 2^{m} r(E)\right)$. Using the doubling property, we get

$$
\begin{aligned}
\mu(B) & \leq \mu\left(B\left(x(E), 2^{m} r(E)\right)\right) \\
& \leq A^{m} \mu(B(x(E), r(E)))=A^{m} \mu(E) .
\end{aligned}
$$

Now the second statement of (i) follows with any $0<\alpha<A^{-m}$.

Proof of Theorem 1] Let the linear space $X$ be infinite dimensional. Let $B=$ $B(0,1)$ be the unit ball in $X$. Then by F. Riesz' theorem the ball $B$ is not compact (Theorem 9.2 in 9]). Because $X$ is complete this means that $B$ is not totally bounded. Therefore, there exists $\epsilon>0$ and at least a countable family of disjoint balls $B_{j}=B\left(x_{j}, \epsilon\right) \subset B$, all contained in $B$. One can see the existence of a disjoint family by looking at the cover of a unit open parallelogram by a family of its $2 \epsilon$ multiples, in which case they can be fitted together to produce a disjoint cover. According to Theorem 2 there exists a constant $C>0$ such that $\mu\left(B_{j}\right) \geq C \mu(B)$. Because all $B_{j}$ are disjoint, it follows that $\mu(B)$ must be infinite, a contradiction with the assumption $\mu(B)<\infty$.

\section{ACKNowledgement}

I would like to thank Professor Guozhen Lu for drawing my attention to the subject and for suggesting the problems. I would also like to thank him and Professor Steen Pedersen for many valuable discussions during my visit to the Wright State University in May 1999. 


\section{REFERENCES}

[1] B. Franchi, G. Lu and R.L. Wheeden, 'A relationship between Poincaré-type inequalities and representation formulas in spaces of homogeneous type', Internat. Math. Res. Notices 1 (1996) 1-14. MR 97k:26012

[2] Y. Liu, G. Lu and R.L. Wheeden, 'Some equivalent definitions of high order Sobolev spaces on stratified groups and generalizations to metric spaces', preprint.

[3] Y. Liu, G. Lu and R.L. Wheeden, 'Representation formulas and Sobolev spaces of high order on stratified groups and generalizations to metric spaces', Math. Sci. Res. Hot-Line 3 (1999), 35-59. MR 2000f:46044

[4] G. LU, 'Local and global interpolation inequalities on the Folland-Stein Sobolev spaces and polynomials on stratified groups', Math. Res. Lett. 4 (1997) 777-790. MR 99i:46024

[5] G. Lu, S. Pedersen and M. Ruzhansky, 'Distribution theory, high order gradients and polynomials on metric spaces', in preparation.

[6] G. Lu and R.L. Wheeden, 'Poincaré inequalities, isoperimetric estimates, and representation formulas on product spaces', Indiana Univ. Math. J. 47 (1998) 123-151. MR 99e:46043

[7] G. Lu and R.L. WheEden, 'High order representation formulas and embedding theorems on stratified groups and generalizations', to appear in Studia Math.

[8] N. OватA, White noise calculus and Fock space (Springer, Berlin, New York, 1994). MR 96e:60061

[9] F. Treves, Topological vector spaces, distributions and kernels (Academic Press, New York, London, 1967). MR 37:726

Department of Mathematics and Statistics, University of Edinburgh, Mayfield Road, Edinburgh EH9 3JZ, United Kingdom

E-mail address: ruzh@maths.ed.ac.uk

Current address: Department of Mathematics, Imperial College, 180 Queen's Gate, London SW7 2BZ, England

E-mail address: ruzh@ic.ac.uk 